\title{
Paul de Lagarde und Titus von Bostra
}

\author{
Zur manichäologischen Forschung vor der Entdeckung der \\ manichäischen Selbstzeugnisse
}

Erst seit dem Beginn des 20. Jh.s liegen uns manichäische Selbstzeugnisse vor. Diese wurden zunächst im Zuge der deutschen, britischen, russischen, japanischen und französischen archäologischen Expeditionen an die Seidenstrassen geborgen. Das umfangreichste Material bildeten dabei die Handschriftenfunde im Zuge der vier preußischen resp. deutschen Expeditionen nach Ost-Turkistān aus den Jahren 1902-1914 (die bekannten „Turfan-Expeditionen“ - wenngleich diese die Teilneh mer auch zu anderen historischen Stätten führten). Bei diesen Kampagnen wurden Bruchstücke manichäischer Handschriften vor allem in uigurischer, sogdischer, mittelpersischer und parthischer Sprache aufgefunden, ${ }^{1}$ daneben jedoch auch Bildwerke der zentralasiatischen Manichäer entdeckt. ${ }^{2}$ Durch

1 Vgl. hierzu die Überblicksdarstellungen Lieu, Samuel N. C.: Manichaeism in Central Asia and China. Leiden 1998 (Nag Hammadi and Manichaean Studies 45) und Sundermann, Werner: Der Manichäismus an der Seidenstraße. Aufstieg, Blüte und Verfall. In: Die Seidenstraße. Handel und Kulturaustausch in einem eurasiatischen Wegenetz. Hrsg. v. Ulrich Hübner [u. a.]. 2. Aufl. Hamburg 2005 (Asien und Afrika: Beiträge des Zentrums für Asiatische und Afrikanische Studien (ZAAS) der Christian-Albrechts-Universität zu Kiel 3). S. 153-169; zu den Turfan-Expeditionen allgemein vgl. Grünwedel, Albert: Bericht über archäologische Arbeiten in Idikutschari und Umgebung im Winter 1902-1903. München 1906 (Abhandlungen der Bayerischen Akademie der Wissenschaften, I. Kl. XXIV, Bd. I, I. Abt.), Ders.: Altbuddhistische Kultstätten in Chinesisch Turkistan. Bericht über archäologische Arbeiten von 1906 bis 1907 bei Kuča, Qarašahr und in der Oase Turfan. Berlin 1912 und v. Le Coq, Albert August: Bericht über Reisen und Arbeiten in Chinesisch-Turkistan. In: Zeitschrift für Ethnologie 39 (1907). S. 509-524, Ders.: A short account of the origin, journey, and results of the First Royal Prussian (Second German) expedition to Turfan in Chinese Turkistan. In: Journal of the Royal Asiatic Society (1909). S. 299-322, Ders.: Die vierte deutsche Turfanexpedition. In: Túrán 1 (1918). S. 7-24 etc.

2 v. Le Coq, Albert August: Die buddhistische Spätantike in Mittelasien II. Die manichäischen Miniaturen. Berlin 1923, Heuser, Manfred u. Hans-Joachim Klimkeit: Studies in Manichaean literature and art. Leiden 1998 (Nag Hammadi and Manichaean Studies 46), Gulácsi, Zsuzsanna: Manichaean art in Berlin collections. A comprehensive catalogue of Manichaean artifacts belonging to the Berlin State Museums of the Prussian Cultural Foundation, Museum of Indian Art, and the Berlin-Brandenburg Academy of Sciences, deposited in the Berlin State Library of the

Ә OpenAccess. ( 2020 Michael Knüppel, publiziert von De Gruyter. (cc)BY Dieses Werk ist lizenziert unter der Creative Commons Attribution 4.0 International. https://doi.org/10.1515/9783110615463-007 
die von anderen Mächten ausgesandten Expeditionen konnten zudem noch weitere chinesische Manichaica geborgen werden.

$\mathrm{Zu}$ den Entdeckungen in Ost-Turkistān traten später noch die Funde aus dem Niltal - so im Jahre 1929, als sieben koptische Codices im ägyptischen Medinet Madi gefunden wurden (hierunter Briefe Manis, der sogenannte Synaxis-Codex, Homilien, Teile einer manichäischen Kirchengeschichte, die „Kephalaia des Lehrers“, die „Kephalaia der Weisheit meines Herrn Mani“ sowie das manichäische Psalmenbuch). ${ }^{3}$ Die Liste der Funde, die sich bis zum heutigen Tage fortsetzen ließe und so bedeutende Stücke wie den 1969 erworbenen „Kölner Mani Codex" umfasst, könnte an dieser Stelle ebenso weitergeführt werden, wie sich Ausführungen zur Editionsarbeit an den Schriftzeugnissen der Manichäer anbringen ließen. Bis 1902 stellten freilich die Antimanichaica die wichtigsten Quellen für unsere Kenntnis der im 3. Jh. gestifteten „Religion des Lichts“ dar.

Im vorliegenden Beitrag wendet sich der Vf. der Edition eines solchen Textes, Gegenstand des Schaffens P. de Lagardes, zu - der Bearbeitung der anti-mani-

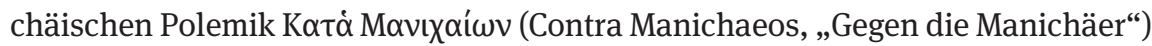

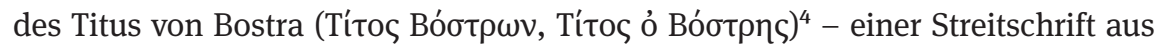
dem 4. Jh. Die Bearbeitung aus den Händen Lagardes wird zwar stets im Zusammenhang mit den manichäologischen Studien vor der Entdeckung manichäischer Selbstzeugnisse und der Untersuchung christlicher Antimanichaica erwähnt ${ }^{5}$ - eine wissenschaftshistorische Einordnung erfolgte hierbei jedoch

Prussian Cultural Foundation. Turnhout 2001 (Corpus Fontium Manichaeorum. Series archaeologica et iconographica 1), Dies.: Mediaeval Manichaean book art. A codicological study of Iranian and Turkic illuminated book fragments from $8^{\text {th }}-11^{\text {th }}$ century East Central Asia. Leiden 2005 (Nag Hammadi and Manichaean Studies 57) etc.

$3 \mathrm{Zu}$ den kopt. Manichaica sowie dem Manichäismus in Ägypten vgl. - neben den Einleitungen zu den diversen Texteditionen - z.B. Richter, Siegfried G.: The Coptic Manichaean library from Madinet Madi in the Fayoum. In: Christianity and monasticism in the Fayoum oasis. Essays from the 2004 International Symposium of the Saint Mark Foundation and the Saint Shenouda the Archimandrite Coptic Society in Honor of Martin Krause. Hrsg.v. G. Gabra. Cairo u. New York 2005. S. 71-78, Ders.: Arbeiten am koptisch-manichäischen Psalmenbuch. Teil 1: Ein Zwischenbericht. In: Il Manicheismo. Nuove prospettive della ricerca. Quinto congresso internazionale di studi sul Manicheismo. Hrsg.v. Aloïs van Tongerloo u. Luigi Cirillo. Turnhout 2005 (Manichaean Studies 5). S. 343-354.

4 Lagarde, Paul de: Titi Bostreni quae ex opere contra Manichaeos edito in codice hamburgensi servata sunt graece e recognitione Paul Antonii de Lagarde. Berlin 1859, Ders.: Titi Bostreni contra manichaeos libri quatuor. Syriace. Berlin 1859.

5 So nennt N. A. Pedersen ihn als den bis dahin wichtigsten Bearbeiter der Polemik des Titus von Bostra (Pedersen, Nils Arne: Demonstrative proof in defence of God. A study of Titus of Bostra's Contra Manichaeos. The work's sources, aims and relation to its contemporary theology. Leiden 2004 (Nag Hammadi and Manichaean studies 56). S. 76). 
nicht (sieht man einmal von den Ausführungen N. A. Pedersens zur Geschichte der Titus-Forschung, die allerdings nicht in erster Linie Lagarde und dessen Textedition [die natürlich Berücksichtigung finden] zum Gegenstand haben, $\mathrm{ab}^{6}$ ).

\section{2}

Über den Vf. der Streitschrift, Titus von Bostra, ist nur sehr wenig bekannt. ${ }^{7}$ Er lebte im 4. Jh. und hat als Bischof von Bostra (= Bosra, arab. Bușrā, Buṣrā aš-Šām) einer im Südwesten Syriens gelegenen Stadt, die zeitweilig die Hauptstadt des Nabatäerreichs war, gewirkt. Die Stadt Bostra war im Zuge der Eroberung durch Trajan in das Römische Reich eingegliedert worden und ab 106 Hauptstadt der Provinz Arabia Petraea (unter römischer Herrschaft Nova Traiana Bostra). Die Wirkungsstätte des Titus bildete zudem eine wichtige wirtschaftliche Schnittstelle, an der sich mehrere Handelsstrassen zum Roten Meer kreuzten. Seit der Mitte des 3. Jh.s war die Stadt eine bedeutende christliche Metropole, die ihre Stellung erst infolge der arabischen Eroberung im Jahre 634 wieder verlor.

Aufgrund der Bedeutung der Stadt als Handelszentrum einerseits und andererseits des Umstandes, dass gerade hier die anti-manichäische Streitschrift des Titus entstand, kann man vermuten, dass in der Stadt im 4. Jh. eine manichäische Gemeinde bestanden hat.

Im Jahre 362 geriet Titus in einen Konflikt mit Kaiser Julian Apostata, an welchen er sich zu dieser Zeit in einem Brief richtete. ${ }^{8}$ Es mag vielleicht auch die Auseinandersetzung zwischen den Christen und den Anhängern der „röm. Religion“ hierbei eine Rolle gespielt haben. Wir wissen über Titus weiterhin, dass er im folgenden Jahr, im Herbst, an der Synode von Antiochia teilnahm.

Seine Streitschrift gegen die Manichäer ist spätestens am 17. Februar 364 (terminus ante quem) fertiggestellt worden, worüber wir durch den Kirchenvater Sophronius Eusebius Hieronymus unterrichtet sind. Einen terminus post quem liefert uns Titus selbst - dieser erwähnt ein Erdbeben, welches sich am 2. Dezember 362 ereignet hat. Die Schrift erlangte wohl früh eine gewisse Bekanntheit so wird sie bereits im „Panarion des Epiphanios“ im Jahre 377 erwähnt. ${ }^{9}$ Wie uns

6 Pedersen, Proof (wie Anm. 5), S. 69-78.

7 Vgl. zu Titus von Bostra Casey, Robert Pierce: Titus von Bostra. In: Pauly-Wissowa-Kroll, Realencyclopädie der classischen Altertumswissenschaft II. Reihe 6. Stuttgart 1937. S. 1586-1591. 8 Der Kaiser hatte sich zuvor in einem Brief an die Einwohner Bostras gewandt und diese aufgefordert, Titus zu vertreiben (Pedersen, Proof (wie Anm. 5), S. 3-4).

9 N. A. Pedersen gibt als möglichen Entstehungszeitraum die Jahre 363 bis 377 an (Pedersen, Proof (wie Anm. 5), S. 1). 
Hieronymus in seinem „De viris inlustribus“ mitteilt, ist Titus in der Zeit der Herrschaft des Kaisers Valens (d. h. zwischen dem 28. März 364 und dem 9. August 378) verstorben.

\section{3}

Der griech. Original-Text der vier Bücher der Streitschrift ist nur teilweise erhalten. Bis 1928 waren nur die beiden ersten Bücher sowie der Anfang des dritten Buches bekannt. In jenem Jahre konnten weitere Teile identifiziert werden. Diese wurden 1973 veröffentlicht resp. von P. Nagel in seiner Habilitationsschrift aus dem Jahre 1967 behandelt. Der syrische Text, der vollständig vorliegt, war hingegen im 19. Jh., also zur Zeit der Bearbeitung und kritischen Edition durch Lagarde (1859), bereits verfügbar. ${ }^{10}$ Vom griech. Urtext existieren nur insgesamt sieben Hss. Die früheste von diesen ist eine Athos-Hs. aus dem 11. Jh. ${ }^{11}$ Einige weitere griech. Parallelen zum syr. Text finden sich in Gestalt von Zitaten in einem Johannes von Damaskus zugeschriebenen Werk. Die älteste Hs. des syr. Textes datiert aus dem November des Jahres 411 - eine syr. Übersetzung wurde also offenbar nur wenige Jahre oder Jahrzehnte nach dem Bekanntwerden des griech. Textes angefertigt. Von der syr. Fassung existiert nur eine Hs., die sich heute in den Beständen der British Library befindet.

Zwar hatte der Text schon vor der Mitte des 19. Jh.s immer wieder die Aufmerksamkeit der Theologen gefunden - zu nennen sind hier etwa die häresiologischen Schriften, in denen bereits im 16. Jh. protestantische Theologen als „Manichäer“ verteufelt wurden, und hierbei auch auf antimanichäische Streitschriften (darunter auch jene des Titus von Bostra) zurückgegriffen wurde ${ }^{12}$-, es sollte jedoch dem in Göttingen wirkenden Paul de Lagarde vorbehalten bleiben, die erste Edition der damals bekannten griech. und syr. Fassungen dieser bedeutenden Sekundärquelle zu Manichäismus vorzulegen.

10 Das unvollständige griechische Manuskript, das heute zumeist als „Hamburger Manuskript“ bekannt ist, liegt seit dem 18. Jh. der Forschung vor.

11 Einen Überblick, unter Berücksichtigung der Verhältnisse der Handschriften zueinander geben Roman, Agathe / Schmidt, Thomas S. / Poirier, Paul-Hubert / Crégheur, Éric / Declerck, José H.: Titi Bostrensis Contra Manichaeos libri IV. Graece et syriace. Textvm graecvm librorum I-III, 30 A. Turnhout 2013 (Corpvs Christianorvm. Series Graeca 82). S. XXII ff.

12 Vgl. hierzu ausführlich Pedersen, der in seinem wissenschaftshistorischen Abriss diese Vorgeschichte bis zur Edition durch de Lagarde zusammenfasst (Pedersen, Proof (wie Anm. 5), S. 69-76). 
Die frühesten Bearbeitungen des Titus-Textes waren Übersetzungen ins Lateinische, welche im 17. Jh. entstanden waren. Erst 1725 wurde von J. Basnage die Editio princeps des erhaltenen griech. Textes herausgebracht. Der Edition durch Basnage war das „Hamburger Manuskript“ zugrunde gelegt worden. Diese fand, nach einer Neuauflage im Jahre 1769 weitere Aufmerksamkeit. Lagarde war der erste, der die zwischen den Fassungen bestehenden Interpolationen erkannte. ${ }^{13}$

\section{4}

Für die Einschätzung der Bedeutung der auch für neuere Editionen herangezogenen ${ }^{14}$ Bearbeitung des Titus von Bostra durch Lagarde für die manichäischen Studien ist es zunächst erforderlich, einen Blick auf die Forschungsgeschichte vor der Entdeckung der ersten manichäischen Selbstzeugnisse allgemein, aber auch bis zum Wirken Lagardes und auf die „manichäologische Forschungslandschaft“ in der Mitte des 19. Jh.s im Besonderen zu werfen. Die Darstellung kann an dieser Stelle etwas gestrafft gegeben werden - findet sich ein forschungsgeschichtlicher Abriss doch schon bei N. A. Pedersen. ${ }^{15}$

Weithin umstritten ist die Frage danach, mit welchen Gelehrten die Anfänge der wissenschaftlichen Manichäologie zu verbinden sind. Zu Lagardes Zeit waren die diversen Antimanichaica - von den Schriften des Hl. Augustinus, über Johannes von Damaskus, Titus von Bostra, Alexander von Lycopolis bis hin zu Serapion von Thumis seit Jahrhunderten ebenso bekannt, wie die Ketzerkataloge der Kirchen in Ost und West. Natürlich könnte man in jeder Bezugnahme auf diese oder in jedem Zitat - etwa aus den antimanichäischen Schriften des Hl. Augustinus - den Beginn der Manichäologie erblicken. Eine ausdrückliche Auseinandersetzung mit der „Religion des Lichts“ freilich ist in solchen Zitaten und Bemerkungen nicht auszumachen - auch entsprach die Intention der christlichen

13 Zur Arbeit de Lagardes an Titus' Text aufschlussreich sind seine diesbezüglichen Notizen, die im de Lagarde-Nachlass in den Beständen der Abteilung Handschriften und Seltene Drucke der Niedersächsischen Staats- und Universitätsbibliothek zu Göttingen bewahrt sind (vgl. hierzu Nachlaß von Paul Anton de Lagarde. In: Verzeichniss der Handschriften im preussischen Staate. I: Hannover, 3: Göttingen, 3: Universitätsbibliothek: Nachlässe [...]. Berlin 1894. S. 133-153, hier S. 140, Lagarde 53-55).

14 Pedersen, Proof (wie Anm. 5); Roman / Schmidt / Poirier / Crégheur / Declerck, Titi Bostrensis (wie Anm. 11); Roman, Agathe / Schmidt, Thomas S. / Poirier, Paul-Hubert: Contre les manichéens. Titus de Bostra. Introd., trad., notes et index. Turnhout 2015 (Corpvs christianorvm in translation 21; Corpvs christianorvm 82).

15 Pedersen, Proof (wie Anm. 5), S. 69-78. 
(und anderen) Antimanichaica nicht einer Beschreibung aus dem Bestreben um einen Erkenntnisgewinn heraus.

So ist es denn auch schwierig, den „Vater der Manichäologie“ eindeutig zu benennen. Ein gelegentlich als solcher betrachteter Gelehrter war der protestantische Theologe Cyriacus Spangenberg (7.6.1528-10.2.1604), der sich in seiner Schrift Apologia Bericht vnd Erklerung M. Cyria[ci] Spangenberg gegen den Vorwurf, Manichäer zu sein, verteidigte. ${ }^{16}$ Seine Arbeit freilich ist ebenfalls kein um wissenschaftliche Erkenntnis hinsichtlich der Religion des persischen Religionsstifters bemühter Beitrag, sondern eine Verteidigungsschrift. Ein anderer Autor, der als „Anwärter“ auf den Titel des Begründers der Manichäologie ins Spiel gebracht werden kann, war der spanische Jesuit Francisco Torres (um 1504/ 1509-8.11.1584), der in seine Übersetzung einer griech. Haeresiographie auch die Polemik des Titus einbezog. Sein Werk blieb allerdings bis über seinen Tod hinaus unpubliziert und wurde erst 1604 von H. Canisius gedruckt. ${ }^{17}$ Das Werk von Torres hatte allerdings ebenfalls kein Bemühen um Erkenntnis zum Gegenstand, sondern gehörte in den oben bereits angesprochenen Kontext der katholisch-protestantischen Polemik, für die Haeresiographien der älteren Kirche instrumentalisiert wurden. Problematisch ist daher auch die Benennung des protestantischen Theologen Isaac de Beausobre (8.3.1659-5.6.1738), der zwar erstmals alle ihm zugänglichen Informationen zu Manis Lehre zusammentrug und wegen seines Hauptwerkes Histoire Critique de Manichée et du Manichéisme (1734 u. 1739) ${ }^{18}$ häufiger auch als der Begründer der manichäischen Studien betrachtet wurde, ${ }^{19}$ allerdings hatte dieser sein Werk mit der Zweckbestimmung der Deutung

16 Spangenberg, Cyriacus: Apologia. Bericht vnd Erklerung M. Cyria[ci] Spangenberg: Der Sieben Predigten halben, von der Praedestination: Göttlichen Versehung, vnd Ewigen Gnadenwahl in Druck geben. Eisleben 1568; zu C. Spangenberg vgl. Kaufmann, Thomas: Spangenberg, Cyriacus. In: Neue Deutsche Biographie 24. München 2010. S. 623-624.

17 Canisius, Henricus: Antiquæ lectionis. Tomus V: Bipartitus, in quo L. vetera monumenta, nunquam visa, pauculis exceptis. Quorum Catalogum versa pagina indicat. Omnia nunc primum e manuscriptis edita et notis illustrata / ab Henrico Canisio Noviomago ic. et ss. canonum professore ordinario in Academia Ingolstadiensi. Ingolstadii 1604.

18 De Beausobre, Isaac: Histoire Critique de Manichée et du Manichéisme. 2 Bde. Amsterdam $1734-1739$.

19 Rudolph, Kurt: Berlin als Zentrum manichäischer Studien seit 250 Jahren. In: Studia Manichaica. IV. Internationaler Kongreß zum Manichäismus, Berlin 14.-18. Juli 1997. Hrsg. v. Ronald E. Emmerick [u.a.]. Berlin 2000 (Berichte und Abhandlungen. Sonderband 4). S. 1-13, Stroumsa, Guy G.: Isaac de Beausobre revisited: The birth of Manichaean studies. In: Studia Manichaica. IV. Internationaler Kongreß zum Manichäismus, Berlin 14.-18. Juli 1997. Hrsg. v. Ronald E. Emmerick [u. a.]. Berlin 2000 (Berichte und Abhandlungen. Sonderband 4). S. 601-612 und van Oort, Johannes: Würdigung Isaac de Beausobres (1659-1738). In: Studia Manichaica. IV. Internationaler 
der „Religion des Lichts“ als eines frühen Protestantismus verfasst und war, wie Spangenberg oder Torres, nicht um eine tatsächliche wissenschaftliche Annäherung an die Lehre Manis bemüht.

Eher schon wird man den Historiker und ersten Kanzler der Georg-AugustUniversität Göttingen, Johann Lorenz von Mosheim (1693-1755), als „Vater der Manichäologie“ ansehen dürfen. ${ }^{20}$ von Mosheim bezog den Manichäismus in seine Arbeiten zur Ketzergeschichte - hier Anderweitiger Versuch einer vollständigen und unpartheyischen Ketzergeschichte, „De rebus christianorum ante Constantinum Magnum commentarii und Institutionum historiae ecclesiasticae antiquae et recentioris libri quatuor" - ein und kontextualisierte die Lehre Manis in diesem kirchengeschichtlichen Gesamtzusammenhang. ${ }^{21}$

Die Darstellungen bei von Mosheim hatten mit denen seiner „Vorläufer“ jedoch gemein, dass sie ausschließlich auf zu dieser Zeit bekannte oder vielmehr zugängliche christliche Quellen gegründet waren. Die Situation sollte sich im 19. Jh. jedoch insofern ändern, als in dieser Zeit allmählich auch die Antimanichaica aus den Ostkirchen und den islamisch geprägten Kulturräumen im Westen bekannt wurden, so z. B. bei Ferdinand Christian Baur (1792-1860). ${ }^{22}$ In der zweiten Hälfte des 19. Jh.s konnten diese aufgrund der Leistungen Baurs in die Betrachtungen/ Untersuchungen einbezogen werden - und es war diese Umbruchsituation, in der die Bearbeitung des Textes des Titus von Bostra durch Lagarde erfolgte.

Kongreß zum Manichäismus, Berlin 14.-18. Juli 1997. Hrsg. v. Ronald E. Emmerick [u.a.]. Berlin 2000 (Berichte und Abhandlungen. Sonderband 4). S. 658-666.

20 Wesseling, Klaus-Gunther: Mosheim, Johann Lorenz. In: Biographisch-Bibliographisches Kirchenlexikon 6: Moenius, Georg - Patijn, Constantijn Leopold. Herzberg am Harz 1993. Sp. 196204, hier Sp. 199-204.

21 Mosheim, Johann Lorenz v.: Versuch einer unpartheyischen und gründlichen Ketzergeschichte. Helmstaedt 1746, Ders.: De rebus christianorum ante Constantinum Magnum commentarii. Helmstedt 1753 [engl. Übers. v. J. Murdock: Historical commentaries on the state of Christianity during the first three hundred and twenty-five years from the Christian era; being a translation of the commentaries on the affairs of the Christians before the time of Constantine the Great. 2 Bde. New York 1852], Ders.: Institutionum historiae ecclesiasticae antiquae et recentioris libri quatuor. Helmstedt 1755.

$22 \mathrm{Zu}$ Ferdinand Christian Baur vgl. Bautz, Friedrich Wilhelm: Baur, Ferdinand Christian. In: Biographisches-Bibliographisches Kirchenlexikon 1. Herzberg am Harz 1975. coll. 427-428, Scholder, Klaus: Baur, Ferdinand Christian. In: Theologische Realenzyklopädie 5. Hrsg. v. Gerhard Müller [u. a.]. 1. Aufl. Berlin 1980, S. 352-359. 
Das Interesse Lagardes, der später auch mit anderen Manichäologen (z.B. C. Kessler) in Verbindung stand, ${ }^{23}$ galt allerdings nicht der Lehre der persischen Religionsstifters an sich. Lagarde hat sich außerhalb der Bearbeitung des Textes Titus von Bostras ohnehin nicht mit dem Manichäismus befasst - von Korrespondenzen einmal abgesehen - und stand einer Annäherung an diesen Forschungsgegenstand (wie überhaupt religionswissenschaftlichen Fragestellungen im Sinne einer historisch-vergleichenden Betrachtung [unter Einbeziehung nichtabrahamitischer Religionen]) wohl eher ablehnend gegenüber. Lagarde sah sich ja bekanntlich nicht als Orientalist und ist nur unter Vorbehalt als Manichäologe $\mathrm{zu}$ betrachten - das Interesse an Titus von Bostra und seinem Werk entsprang vielmehr seinen Studien zur syrischen Kirchengeschichte. ${ }^{24}$ Bereits 1852 hatte sich Lagarde für die Befassung mit dem Alt-Syrischen nach London, wo er in der British Library syrische Hss. studierte und Abschriften syrischer Texte zur älteren Kirchengeschichte anfertigt hat, begeben. Im folgenden Jahr folgte ein Aufenthalt gleicher Zweckbestimmung in Paris. Sein Interesse für die syrische Kirchengeschichte mag z.T. auch die spärliche Kommentierung des von ihm bearbeiteten Textes erklären. Auf den Betrachter unserer Tage wirkt diese geradezu enttäuschend und es stellt sich zwangsläufig die Frage, warum sie - trotz der Zeitstellung des Werkes Lagardes (die Arbeit F. C. Baurs ${ }^{25}$ lag bereits vor!) so dürftig ausfällt.

Das Desinteresse des Bearbeiters an der „Religion des Lichts“ war freilich nur einer der Gründe hierfür, ein anderer ist zweifelsohne in den philologischen

23 Vgl. beispielsweise das Schreiben P. de Lagardes an Kessler vom 30.3.1889 (in einer Abschrift Anna de Lagardes, in den Beständen der Abteilung Handschriften und Seltene Drucke der Niedersächsischen Staats- und Universitätsbibliothek Göttingen, - dort unter Signatur „Cod. Ms. Lagarde $150: 624^{\prime}$ ) - einem Schreiben in dem sich de Lagarde für die Zusendung des manichäologischen Werkes Kesslers aus demselben Jahr bedankt. Dass die sehr wenigen Korrespondenzen de Lagardes, in denen manichäologische Gegenstände berührt werden, allesamt aus späterer Zeit stammen, war kein Zufall, sondern vielmehr der Persönlichkeit des „wissenschaftlichen Einzelgängers“ de Lagarde, der von der Angst vor dem Diebstahl seines geistigen Eigentums geradezu besessen war, geschuldet. De Lagarde tauschte sich während der Bearbeitung des Textes nicht mit Kollegen über die Inhalte (auch jenseits des Umstandes, dass diesen kaum seine Aufmerksamkeit galt) - vor allem die möglichen religionshistorischen Aspekte - aus.

24 Hierzu ausführlich Göttinger Arbeitskreis für Syrische Kirchengeschichte (Hrsg.): Paul de Lagarde und die syrische Kirchengeschichte. Mit einem Geleitwort von Hermann Dörries. Göttingen 1968.

25 Baur, Ferdinand Christian: Das manichäische Religionssystem nach den Quellen neu untersucht und entwickelt. Tübingen 1831 [Neudrucke: Göttingen 1928. Hildesheim u. New York 1973]. 
Editionsprinzipien der orientalistischen Disziplinen, die sich z.T. im 19. Jh. erst als eigenständige Forschungsrichtungen herausdifferenzierten, zu erblicken (- sie waren ja zumeist „Ableger“ der Theologie [und seltener auch der Geschichtswissenschaften]). Häufig wurden die Realien vernachlässigt und es ging primär um die bloße Übersetzung als eigentlichem Ziel der Editionsarbeit. Das Verständnis - oder vielmehr die inhaltliche Kontextualisierung - des Textes stand demgegenüber häufig zurück - Lagarde bildete in dieser Beziehung keine Ausnahme. Hinzu trat weiterhin, dass eine andere Intention bei der Edition von Texten - das Bemühen um das Verständnis der gerade erst neu entdeckten Sprachen - im konkreten Fall ebenfalls entfiel: das Syrische und Griechische waren zu dieser Zeit längst bekannt und es versprach die weitergehende Befassung mit dem Text keinen Erkenntnisgewinn (soweit sich Lagarde denn im konkreten Fall, d.h. in Bezug auf die Religion Manis, mit welcher sich Titus auseinandersetzt, für einen solchen überhaupt interessiert hat). Es stellt sich also die Frage des Wertes der Bearbeitung des Werkes durch Lagarde (- ungeachtet des Umstandes, dass es sich tatsächlich um die bis dahin gründlichste Bearbeitung der Polemik des Titus von Bostra handelte), dessen Arbeit sich auf die Lesung der ihm zugänglichen und bekannten Teile der Hs. des Werkes von Titus von Bostra und den originalsprachlichen Abdruck beschränkte - hier den Wert für Zeitgenossen, die manichäologischen Forschungen und nachfolgende vergleichende Religionswissenschaftler und Manichäologen.

Rückblickend muss man - selbst dann, wenn man die Zeit und die Entstehungsumstände in Rechnung stellt - einwenden, dass der Einfluss der Bearbeitung Lagardes auf die manichäologischen Studien und ihre Entwicklung (ganz im Gegensatz zu dem erwähnten, bereits zuvor erschienenen Werk Baurs oder den wenig später herausgekommenen Beiträgen Flügels ${ }^{26}$ und Kesslers ${ }^{27}$ ) äußerst begrenzt war. Titus von Bostra und seine Schriften erregten (von vereinzelten Bezugnahmen einmal abgesehen) erst wieder im 20. Jh. das Interesse der Forschung - zu einer Zeit also, als die Antimanichaica vor dem Hintergrund der Entdeckung manichäischer Selbstzeugnisse ihre Bedeutung teilweise eingebüßt hatten resp. die Fragen, die an diese Texte gerichtet wurden, gänzlich anderer Natur waren. Nach der Edition der Texte durch Lagarde, blieb das anti-manichäische Werk des Titus von Bostra zunächst ohnehin jahrzehntelang unbe-

26 Flügel, Gustav: Mani, seine Lehre und seine Schriften. Ein Beitrag zur Geschichte des Manichäismus. Aus dem Fihrist des Abû'l-faradsch Muhammed ben Ishạa al-Warrâḳ, bekannt unter dem Namen Ibn Abî Ja ${ }^{c}$ kûb an-Nadîm, im Text neben Uebersetzung, Commentar und Index zum ersten Mal herausgegeben. Leipzig 1862 [Neudruck: Osnabrück 1969].

27 Kessler, Conrad: Mani. Forschungen über die manichäische Religion. Ein Beitrag zur vergleichenden Religionsgeschichte des Orients. I: Voruntersuchungen und Quellen. Berlin 1889. 
rücksichtigt, bis sich beginnend mit den 1920er Jahren eine Vielzahl von Gelehrten (L. Nix ${ }^{28}$ A. Baumstark, ${ }^{29}$ R. P. Casey, ${ }^{30}$ C. R. C. Allberry, ${ }^{31}$ H. H. Schaeder ${ }^{32}$ ) mit dem Werk auseinandersetzten. R. A. Reitzenstein ${ }^{33}$ gibt einen guten Überblick über die Forschungen, die allerdings bis zum Erscheinen seines Beitrages im Jahre 1931 fest keine publizierten Ergebnisse hervorgebracht hatten. ${ }^{34}$ Unter diesen tritt besonders die Übersetzung von Ludwig $\mathrm{Nix},{ }^{35}$ die inzwischen eine interessante Geschichte hat, hervor. ${ }^{36}$ Dessen deutsche Übersetzung sollte die Grundlage für eine vollständige Re-Edition bilden. Allerdings blieb das Ms. unpubliziert und auch die angestrebte Bearbeitung gelangte nicht über Planungen hinaus.

Mit den Bemühungen von C. R. C. Allberry und H. H. Schaeder freilich war der Standort Göttingen wieder in die Forschungen involviert. Dass diese jedoch zu keinen Resultaten gelangten, war wohl vor allem auf den Ausbruch des Zweiten Weltkriegs zurückzuführen. Schaeder hatte das Ms. 1938 erhalten, Allberry, der bereits mit Vorarbeiten zu einer entsprechenden Arbeit begonnen hatte, verstarb am 3.4.1943. Allberrys Vorarbeiten konnten später aber immerhin noch von P.-H.

28 Vgl. hierzu unten.

29 Baumstark, Anton: Der Text der Mani-Zitate in der syrischen Übersetzung des Titus von Bostra. In: Oriens Christianus 4, 3. Serie (1931). S. 23-42, Ders.: Die syrische Übersetzung des Titus von Bostra und das „Diatessaron“. In: Biblica 16 (3) (1935). S. 257-299.

30 Casey, Robert Pierce: The text of the anti-manichaean writings of Titus of Bostra and Serapion of Thumis. In: Harvard Theological Review 21 (1928). S. 97-111.

31 Der brit. Koptologe und Ägyptologe Charles Robert Cecil Augustin Allberry (1911-1943). Zu C. R. C. A. Allberry vgl. Lewis, Patricia K. G.: Charles Allberry. A portrait. Cambridge 1984.

32 Vgl. hierzu unten.

$33 \mathrm{Zu}$ dem Göttinger Religionswissenschaftler und Klassischen Philologen Richard August Reitzenstein (1861-1931) vgl. Fauth, Wolfgang: Richard Reitzenstein, Professor der Klassischen Philologie 1914-1928. In: Die Klassische Altertumswissenschaft an der Georg-August-Universität Göttingen. Eine Ringvorlesung zu ihrer Geschichte. Hrsg. v. Carl Joachim Classen. Göttingen 1989 (Göttinger Universitätsschriften, Serie A: Schriften, Bd. 14). S. 178-196.

34 Reitzenstein, Richard August: Eine wertlose und eine wertvolle Überlieferung über den Manichäismus. In: Nachrichten von der Gesellschaft der Wissenschaften zu Göttingen, Phil.-hist. Kl. (1931). S. 28-58, hier S. 47.

35 Nix, Ludwig: Titi Bostrensis episcopi contra Manichaeos libri quatuor. Aus dem Syrischen übersetzt. Deutsche Akademie der Wissenschaften zu Berlin, Institut für griechisch-römische Altertumskunde, Archiv, Acc. Nr. 625, 2 (handschriftliches Manuskript 1901).

36 Klein, Wassilios W.: Ludwig Nix (†1904). Privatdozent für semitische Sprachen in Bonn, nebst seiner Habilitationsschrift „Die südarabische Sage“ und einem Vorwort von Stephan Conermann. Schenefeld 2007 (Bonner islamwissenschaftliche Hefte 2), Ders.: Der Semitist Privatdocent Dr. Ludwig Nix† (Bonn) und seine Arbeit zu Titus von Bostra. In: Vom Nil an die Saale. Festschrift für Arafa Mustafa zum 65. Geburtstag am 28. Februar 2005. Hrsg. v. A. Drost-Abgarjan [u. a.]. HalleWittenberg 2008 (Hallesche Beiträge zur Orientwissenschaft 42/ 06). S. 171-180. 
Poirier $^{37}$ und N. A. Pedersen ${ }^{38}$ für ihre Arbeiten verwendet werden. Für all diese Unternehmungen war die Arbeit de Lagardes freilich nur eine Episode und für die Manichäologie fast bedeutungslos angesichts der Leistungen Baurs, Flügels und Kesslers sowie des sich grundlegend ändernden Erkenntnisstandes seit der Entdeckung der manichäischen Selbstzeugnisse im Zuge der eingangs erwähnten Expedition nach Ost-Turkistān sowie dem Bekanntwerden der ägyptischen Manichaica.

37 Poirier/ Roman/ Schmidt/ Crégheur/ Declerck, Titi Bostrensis (wie Anm. 11). 38 Pedersen, Proof (wie Anm. 5). 
